\title{
The Hox gene Antennapedia regulates wing development through
}

\section{0-hydroxyecdysone in insect}

6

7 Chunyan Fang ${ }^{1}$, Yaqun Xin ${ }^{1}$, Tao Sun ${ }^{1}$, Antónia Monteiro ${ }^{2,3}$, Zhanfeng Ye ${ }^{1}$, Fangyin

Dai $^{1}$, Cheng Lu ${ }^{1}$, Xiaoling Tong ${ }^{1 *}$

9

${ }^{1}$ State Key Laboratory of Silkworm Genome Biology, Key Laboratory of Sericultural Biology and Genetic Breeding, Ministry of Agriculture and Rural Affairs, Southwest University, Chongqing 400715, China

${ }^{2}$ Department of Biological Sciences, National University of Singapore, 14 Sciences Drive 4, Singapore

${ }^{3}$ Science Division, Yale-NUS College, 10 College Avenue West, Singapore

* Corresponding author

E-mail: xltong@swu.edu.cn (XT) 


\section{Abstract}

A long-standing view in the field of evo-devo is that insect forewings develop without any Hox gene input. The Hox gene Antennapedia (Antp), despite being expressed in the thoracic segments of insects, has no effect on wing development. This view has been obtained from studies in two main model species, Drosophila and Tribolium. Here, we show that partial loss of function of Antp resulted in reduced and malformed adult wings in Bombyx, Drosophila, and Tribolium. Antp mediates wing growth in Bombyx by directly regulating the ecdysteriod biosynthesis enzyme gene (shade) in the wing tissue, which leads to local production of the growth hormone 20E. In turn, 20E signaling also up-regulates Antp. Additional targets of Antp are wing cuticular protein genes $C P G 24, C P H 28$, and $C P G 9$, essential for wing development. We propose, thus, that insect wing development occurs in an Antp-dependent manner. Key words: Hox, Antennapedia, shade, 20E, cuticular protein gene, wing development

\section{Introduction}

The Hox genes encode a family of transcriptional regulators that are important in differentiating the bodies of bilaterian animals along their antero-posterior axis [1]. Disruptions to individual Hox genes often leads to disruptions of traits that develop in the regions where the Hox gene is expressed [1].

In holometabolous insects, the Hox gene Antennapedia (Antp) is expressed in all thoracic segments, including in the forewing and hindwing yet, no function has been attributed to this gene regarding wing morphogenesis. In Drosophila, wing and haltere primordia could be detected in embryos even in the complete absence of Antp function in homozygous mutants of Antp [2]. In addition, a very low level of Antp protein present in the growing wing imaginal disc, suggested that forewing formation did not require Antp [2]. Similarly, no obvious phenotypes were observed in adult Tribolium elytra (forewing) or hindwing after RNA interference (RNAi) of Antp. These data suggested that wing development takes place without any Antp input [3]. In contrast, the Hox gene Ultrabithorax (Ubx), expressed exclusively in hindwings, functions to differentiate hindwings from forewings. Forewing development in insects was, thus, thought to occur without any significant Hox gene input [3-13].

Recently, however, we observed that two loss of function mutations in the silkworm Bombyx mori Antp gene (BmAntp), Nc and Wes, displayed abnormal wings [14,15]. These mutations had not been examined beyond the embryonic stage due to lethality, but could be maintained in heterozygous lines. The adults of these lines displayed reduced and malformed wings.

These two Bombyx Antp mutants, however, shared common features with Drosophila Antp mutants, observable in embryos. The homozygous (Antp ${ }^{-/-}$) embryos died late in embryogenesis but displayed a homeotic transformation of thoracic legs to antenna-like appendages[14-17]. The novel wing phenotypes in Bombyx heterozygote mutants, however, suggested that Antp was affecting wing development, a role not 
previously documented for this gene because embryos died long before the stage when wings start to develop. In the present study, we used wiltype and heterozygotes of the Wes strain $\left(A n t p^{+-}\right)$as the study objects to more fully undertstand the role of Antp in wing development.

\section{Results}

\section{BmAntp is involved in the development of wings in Bombyx}

Since defective adult wings were observed in aberrant Antp Wes and Nc mutants $\left(A n t p^{+/}\right)[14,15]$, we sought to test when in development Antp input was required. We analyzed the expression profile of BmAntp in the forewing and hindwing of wildtype (WT) individuals from the $3^{\text {rd }}$ day of the 5 th instar to the adult stage. qRT-PCR revealed that the expression of BmAntp was maintained at a low level in the larval stage and gradually increased and reached a peak on the $6^{\text {th }}$ day of the pupal stage. Forewings expressed higher levels of Antp relative to hindwings at most times during the pupal stage (Fig 1A). Then, we compared the expression pattern of BmAntp between mutant Wes $\left(A n t p^{+/}\right)$and WT individuals. BmAntp was expressed at a consistent but lower level in the mutants compared to WT controls (Fig 1B).

To evaluate the effects of BmAntp expression levels on wing morphology during development, we dissected the wing discs of Wes $\left(A n t p^{+-}\right)$and WT from the $3^{\text {rd }}$ day of the last larval instar to the wandering stage larva. Wing disc size increased slowly during the larval stage and was not significantly different between Wes mutants $\left(A n t p^{+/}\right)$and WT individuals. Then, during the wandering stage, the wing morphology changed dramatically. The wing discs of Wes mutants $\left(A n t p^{+/-}\right)$were curlier and smaller than those of WT, and finally degenerated to tiny and wrinkled adult wings (Fig 1C).

To confirm the function of BmAntp in wing development, we performed RNAi injections into WT larvae of B. mori. We synthesized dsRNA targeting BmAntp and injected it into larvae on the $1^{\text {st }}$ day of the wandering stage. qRT-PCR showed that BmAntp dsRNA efficiently reduced BmAntp transcript levels compared to controls injected with EGFP dsRNA (Fig 1D). Nineteen out of 22 (86\%) BmAntp dsRNA treated individuals had small wings similar to the Wes mutant $\left(A n t p^{+/-}\right)$while control silkworm adults grew their wings normally (Fig 1E).

To further confirm the function of BmAntp we performed crisper-Cas9 injections into WT embryos of $B$. mori. We generated a genomic disruption of the BmAntp gene by targeting its first exon using four specific single-guide RNAs (sgRNAs) and the Cas9/gRNA ribonucleoprotein (RNP) delivery system (Fig 1F). After injection, 20 eggs hatched and 18 larvae developed to the adult stage. We found that $61 \%$ of the moths (11 individuals) displayed malformed adult wings (S2 Table, Fig 1G), and confirmed that various insertions and deletions were present at the location targeted by the four sgRNAs (Fig 1H). Abnormal wings were not observed in control injections with BmBLOS2 sgRNA which only led to translucent larval skin. These data indicate that BmAntp is a critical transcription factor that regulates wing development in B. mori. 


\section{BmAntp affects the synthesis of $20 \mathrm{E}$ by regulating the expression of Shade in wing dises}

We next tested whether the production of abnormal wings in BmAntp mutants was related to deficits in levels of the molting hormone, 20-hydroxyecdysone (20E). We tested this hypothesis because 1) Significant differences in the size of wing discs were observed between BmAntp mutants and controls starting from the onset of the larva-to-pupa transition (Fig 1C); 2) A pulse of this steroid hormone normally regulates the larva-to-pupa transition; and 3) $20 \mathrm{E}$ is a major regulator of wing growth and development $[16,17]$.

We first examined the expression level of genes involved in the ecdysteriod biosynthesis pathway in a variety of tissues. We found that spookier, phantom, disembodied, and shadow were expressed in the prothoracic gland (PG), as expected, as this is the main source of ecdysteroid synthesis in insect larvae [5].In addition, the shade gene, which codes for a P450 monooxygenase that catalyze ecdysone into the active $20 \mathrm{E}$ in targeted peripheral tissues [18], was primarily expressed in the wing discs compared to the PG and hemolymph (Figs 2A-2E).

We next explored whether Wes mutants $\left(A n t p^{+/-}\right)$expressed shade at different levels relative to WT wings, and whether this impacted levels of $20 \mathrm{E}$ in the wing tissue. The shade transcripts were present at markedly higher levels in WT than in Wes mutant $\left(\right.$ Antp $\left.^{+/}\right)$wings, and levels reached a peak on the $4^{\text {th }}$ day of the pupal stage (Fig 2F). Titers of ecdysone measured from wing discs on that day (P4), were similar between Wes mutants $\left(A n t p^{+/}\right)$and WT individuals. Titers of 20E, however, were significantly lower in Wes mutants $\left(A n t p^{+/-}\right.$) relative to WT individuals two days later (on P6) (Figs $2 \mathrm{G}$ and $\left.2 \mathrm{G}^{\prime}\right)$.

We next investigated whether the expression levels of Ecdysone Receptor (EcR) and ultraspiracle (usp) [19], the receptors that bind 20E to transduce edysone signaling to the nucleus were also different between Wes and WT individuals. This is because 20E signaling is known to up-regulate expression of $E c R$ and $u s p$ in the wings of Drosophila [20,21]. Significantly lower levels of usp, and of the two isoforms of $E c R, E c R A$ and $E c R B$ mRNA were detected in the mutants compared with WT on day P4 (S1 Fig). These results suggest that Antp is also regulating the expression of these genes, either directly or indirectly. The latter mechanism could involve Antp up-regulating shade, which increases $20 \mathrm{E}$ titers in the wing cells which, in turn, up-regulates $E c R$ and $u s p$ transcription in wings.

We next sought to test whether shade was a direct target of BmAntp. We examined a $2 \mathrm{~kb}$ region of DNA immediately 5' of the start site of shade for possible Antp binding domains and found a total of five such domains (Figs $2 \mathrm{H}$ and $2 \mathrm{H}^{\prime}$ ). To evaluate the extent that DNA containing one or more of these domains could regulate flanking gene expression we cloned different sized fragments, containing a different number of Antp binding domains, upstream of the reporter gene luciferase. We transfected this plasmid into BmN cells and co-transfected BmAntp in these cells as well (S2 Fig). The largest fragment ( -1985 to -300), containing all five Antp binding sites, led to significantly increased luciferase activity compared to the other four fragments (Fig 2I). These data suggest either that a regulatory region -1985 to -1470 
containing a key Antp binding site or, more likely, that all Antp sites together are required for the transcriptional regulation of shade, and that shade is likely a direct target of BmAntp.

To determine whether BmAntp protein could directly bind to the in silico identified Antp binding sites of the shade promoter, we designed a specific biotinylated probe covering the -1985 to -1470 genomic region of shade and conducted electrophoretic mobility shift assay (EMSA) (Fig 2J). We further validated the direct regulation of BmAntp on Shade transcription through in vivo ChIP-PCR following the BmN cells which were overexpression of FLAG-tagged BmAntp (Fig 2K, S3 Fig). Our data indicated that BmAntp activates the transcription of shade by directly binding to the tested genomic region.

\section{BmAntp is upregulated by $20 \mathrm{E}$}

We next sought to investigate which genes could be driving Antp expression in the wings of B.mori. Antp levels were low throughout wing disc development until the pupal stage, and then followed a slow rise and fall. Because this expression profile resembled the 20E titer profile in B.mori hemolymph [22], we decided to investigate whether the 20E/EcR/USP complex could be upregulating Antp in pupal wings. We first examined potential Ecdysone Response Element (EcRE) binding sites for the complex within the $\sim 2 \mathrm{~kb}$ upstream of BmAntp (counting from the start codon of 5 , UTR) and discovered three such sites (EcRE1, -139- -153 nt; EcRE2, -1034- -1048 nt; EcRE3, -1592- -1606 nt) (Fig 3A). Then, we cloned this $\sim 2 \mathrm{~kb}$ genomic region of BmAntp in front of the luciferase reporter gene and transfected this plasmid into BmN cells, followed by $20 \mathrm{E}$ treatment. Subsequent dual luciferase reporter assays revealed that this region drove significantly higher luciferase activity after 20E application (Fig $3 \mathrm{~B})$. To further confirm the upregulation of BmAntp expression by $20 \mathrm{E}$, we used qRT-PCR to show that the expression of BmAntp was significantly upregulated both in cultured cells and in wing discs after 20E treatment (Figs 3C and 3D). These data show that 20E can upregulate expression of BmAntp via the direct binding of the $20 \mathrm{E} / \mathrm{EcR} / \mathrm{USP}$ complex to a 5' upstream region of the gene.

\section{BmAntp directly regulates wing-specific cuticular protein genes}

In order to explore potential additional targets of Antp, besides shade, that might have contributed to the small wings of adult Wes mutants, we investigated the expression of four cuticular proteins with a known expression profile, that matched that of Antp, in both WT and Wes mutants. In particular, expression levels of $\mathrm{CPH} 28$, CPG24, CPG9 peaked at P5, as did expression of Antp (Fig 1B) [20]. CPG11, by contrast, was expressed primarily during the early $5^{\text {th }}$ instar, and was used as a control gene [23]. Previous work has shown that cuticular proteins are major components of insect wings and that both EcR-mediated signaling as well as other transcripton factors regulate their very dynamic and specific expression profiles [24-26]. qRT-PCR analysis showed that the expression levels of $C P H 28, C P G 24, C P G 9$, and $C P G 11$ in Wes $\left(A n t p^{+/}\right)$were remarkably lower than those of WT (Fig 4A). We explored the direct regulation of these four cuticular proteins by Antp by conducting Luciferase 
reporter assays in $\mathrm{BmN}$ cells with candidate genomic regions ( $3 \mathrm{~kb}$ upstream of each gene) containing putative Antp binding sites (Fig 4B). Increasing BmAntp levels in these cells significantly upregulated the transcription of $C P H 28, C P G 24$, and $C P G 9$ (Figs 4C-4F), but not CPG11. A dual-Luciferase assay with $C P H 28$ further showed that BmAntp can directly elevate the expression of $\mathrm{CPH} 28$ (Fig 4G). Moreover, an EMSA and ChIP-PCR essay showed that BmAntp was able to directly bind the in silico identified Antp binding sites in the CPH28 promoter (Figs 4H and 4I, S4 Fig). These results indicate that BmAntp can upregulate the transcription of these three wing cuticular protein genes, and $\mathrm{CPH} 28$ is likely up-regulatd by a direct interaction of Antp with this gene's promoter.

To determine whether $\mathrm{CPH} 28$ is essential for wing development, we knocked it down using RNAi. CPH28-siRNA was injected into 18 pupae, and the same quantity of scrambled siRNA sequence was injected in control animals. Levels of CPH28 decreased significantly in the wing discs $48 \mathrm{~h}$ after $\mathrm{CPH} 28$-siRNA injections relative to control injections (Fig 4J). The ratio of malformed wings reached $80 \%$ after eclosion (Fig 4K, S3 Table). In contrast, all moths in the control group had normal wings (Fig 4K). These results indicate that $\mathrm{CPH} 28$ is required for the generation of normal wings in silkworms.

\section{Antp function in wing development is conserved in Drosophila and Tribolium}

To evaluate whether the function of Antp in wing development is conserved across other insect orders, we examined the wings of adult flies and beetles after Antp down-regulation. In Drosophila we drove expression of Antp RNAi hairpins in larval and pupal wing discs under the control of the nubbin-gal4 (nub-gal4) driver. All individuals in which Antp was knocked down had rudimentary wings that were reduced in size compred to controls (Figs 5A-5D). In Tribolium, we injected Antp/ptl dsRNA during the last larval stage, just before the onset of rapid wing growth [3]. These injections led to lower mRNA levels of Antp/ptl (S5A Fig) and to wrinkled and shortened forewings (elytra) and hindwings (Figs 5E-J, S5B and S5C Figs). Additionally, the uniform mesonotum phenotype observed in the Antp/ptl RNAi adults was consistent with that reported by Tomoyasu and colleagues (Figs $5 \mathrm{~K}$ and 5L, S5D and S5E Figs) [3]. These observations indicate that Antp plays a crucial role in the development of wings in Drosophila and Tribolium. Taken together, these results demonstrate that Antp participates in insect wing development in a conserved manner.

\section{Discussion}

\section{Hox gene Antp is indispensable for wing development}

Limited experiments in previous Drosophila studies, focusing on embryonic and larval stages, likely prevented the identification of Antp's role in later stages of wing development. Fly embryos homozygous for $A n t p^{\mathrm{W} 10}$, a mutation in the Antp sequence, led to normal wing primordia, whereas ectopic expression of Antp in third instar larval wing discs had no effect on larval wing discs morphology [2]. This lack of results is expected as Antp protein was largely absent in the major region of the growing larval 
discs [2]. In the present study, the nub-Gal4 driver was used to drive $U A S$-Antp ${ }^{R N A i}$ expression in fly wing discs. We chose this driver as its expression was first detected in late 2nd instar wing discs and persisted through late pupal wings [27]. This led to a prolonged silencing of Antp expression and to malformed adult wings in Drosophila. Given that the silkworm Antp was also expressed at low levels in larval wings, but at much higher levels in pupal wings, we speculate that there is no requirement for Antp function during the embryo and larval stages, but Antp is important for wing development in the later pupal stages.

Our RNAi experiment in Tribolium castaneum also identified strong wing defects not previously identified with a previous similar RNAi experiment [3]. This previous study only reported variation of mesonotum morphology [3], which was also found in our experiment. We preformed the Antp RNAi experiment twice ( $>250$ individuals) and obtained consistent defective wing morphologies, that were not observed in control animals injected with dsRNA against EGFP. We speculate that the different outcomes of the two experiments may be due to the different dsAntp fragments used. We used two fragments covering a larger region of the Antp gene (922bp) compared to the $535 \mathrm{bp}$ fragment used by Tomoyasu et al.. Based on the present results, we propose that Antp is necessary for wing development in Bombyx, Drosophila, and Tribolium.

Recently, Antp input was found to be required for the development of two novel traits in the wings of the nymphalid butterfly Bicyclus anynana: silver scales and eyespot patterns, in both forewings and hindwings, but only minor wing growth deformities were reported (see Fig 2B in Matsuoka and Monteiro) [28]. It is possible that the role of Antp has shifted from a general wing growth role to a more specialized role in color pattern formation. This might be the case in this species and other nymphalids where Antp expression has been visualized in the eyespots [27,29]. Alternatively, the mosaic disruptions obtained with this crispr-Cas9 experiment were insufficient to uncover a more general role of Antp in wing growth and development. Most interestingly, the effect of Antp on shade expression shoud be investigated in connection to 20E-mediated eyespot size plasticity in this species [27,29].

\section{Bi-directional regulation between Antp and 20E}

We showed that Antp directly binds to the promoters of shade, a gene coding for the last step in the production of the active ecdysteroid, 20E, and that $20 \mathrm{E}$ was produced inside wing tissues from the precursor ecdysone produced in the prothoracic gland [25,30]. The biosynthesis of 20E, the main hormonal regulator or molting and methamorphosis in insects [17,31], is mediated by the Halloween genes, such as spookier, shroud, disembodied, shadow and shade [32]. shade is known to converts ecdysone into 20-hydroxyecdysone (20E) in peripheral organs such as the fat body, midgut and Malpighian tubules [25,30]. As expected, the mRNA coding for Shade mRNA was present at an extremely low level in the prothoracic gland and also in the hemolymph, but at a higher level in wing discs. Given that the mRNA expression of shade in wing discs of Wes $\left(A n t p^{+/}\right)$mutants was significantly lower than that in normal wing discs, this explains the observed lower levels of 20E, but not of ecdysone, 
in the wing tissue of these mutants, and associated wing disc growth disruptions.

In our study, we also found that supplementary 20E up-regulated Antp in both $\mathrm{BmN}$ cells and in developing wing discs. Similar regulation of Hox genes by $20 \mathrm{E}$ have previously been reported in the Drosophila heart, where the expression of $U b x$ and abdominal- $A(a b d A)$, was also activated by ecdysone signaling [33]. So, Antp upregulates $20 \mathrm{E}$ in the wing, and $20 \mathrm{E}$ together with its nuclear co-receptors (EcR and USP) upregulate Antp, EcR and usp expression.

\section{Antp regulates the expression of wing cuticular protein genes}

Cuticular proteins are major components of insect wings and previous studies had already implicated the regulation of these proteins by other Hox genes. A total of 52 cuticular protein genes were detected in silkworm wing discs by expressed sequence tags [23]. The regulation of one these proteins, BmWCP4, was previously shown to be dependent on the co-binding of the Hox gene BmAbd-A with the transcription factor $B m P O U M 2$, in the gene's promoter [34]. In the present study, we focused on investigating wing cuticular protein genes whose expression patterns were largely congruent with that of Antp [31]. We showed that they were remarkably down-regulated in mutant $\left(A n t p^{+/-}\right)$individuals, and that disruptions to one of these proteins impaired wing development. It remains possible, that many more additional Antp targets remain to be described.

Previous studies have assumed that the forewing is a Hox-free wing [3,5,9]. Our data indicated that Antp is crucial for wing development in insects (Fig 6). It does this by directly enhancing transcription of the steroidogenic enzyme gene shade in wings and, thus, controlling the synthesis of an essential growth hormone, 20E, directly in the wing tissue. In turn 20E signaling upregulates Antp expression. Antp also directly regulates the expression of critical cuticular protein genes in both forewings and hindwings. 


\section{Materials and Methods}

Animal Strains. (1) The wild-type (WT) strain DaZao and mutant strain Wes (Antp ${ }^{+/}$) were obtained from the Silkworm Gene Bank of Southwest University, China. Silkworms were reared on mulberry leaves at $25^{\circ} \mathrm{C}$ in $\sim 75 \%$ relative humidity with a 12:12 h (L:D) photoperiod during their entire life. (2) The following fly stocks were used in this study: The WT $y w$ and nub-gal4 enhancer trap lines (BCF391\#) were obtained from Core Facility of Drosophila Resource and Technology. The $U A S-A n t p^{R N A i}$ (THU2760) was supplied by the Tisng Hua Fly Center. The wildtype $y w$ were used as control flies. All individuals were incubated at $25^{\circ} \mathrm{C}$. (3) The Tribolium castaneum GA-1 strain was used in this study. Insects were reared in whole wheat flour containing $5 \%$ brewer's yeast at $30^{\circ} \mathrm{C}$ under standard conditions.

Bombyx cell lines. The Bombyx mori ovary-derived cell line $\mathrm{BmN}$ was cultured at $27^{\circ} \mathrm{C}$ in TC-100 medium (United States Biological) supplemented with $10 \%$ fetal bovine serum (Gibco) and 2\% penicillin/streptomycin (Gibco).

RNA Extraction and qRT-PCR. Total RNA samples were isolated from wing discs, prothoracic glands, hemolymph, BmN cells, and the whole beetles at different time points or under different conditions, using the MicroElute Total RNA kit (Omega) in accordance with manufacturer instructions. The cDNA was synthesized with $1 \mathrm{ug}$ total RNA using the PrimeScript ${ }^{\mathrm{TM}}$ RT Reagent Kit with gDNA Eraser (TaKaRa). qRT-PCR was performed using a qTOWER ${ }^{3} \mathrm{G}$ system (analytikjena) and a qPCR SYBR Green Master Mix (Yeasen). The eukaryotic translation initiation factor 4A (BmMDB probe ID sw22934) was used as an internal reference in Bombyx, and ribosomal protein S3 (rps3) in Tribolium castaneum. All experiments were independently performed with three biological replicates and the results were calculated using the $2-\Delta \Delta \mathrm{CT}$ method. Primers are listed in S1 Table.

RNAi Experiment in Bombyx and Tribolium. The double-strand RNA (dsRNA) of Antp, CPH28, ptl1, ptl2, and EGFP were synthesized using the RiboMAX Large Scale RNA Production System T7 kit (Promega). Approximately $100 \mu \mathrm{g}$ of synthesized dsAntp was injected into the second chest spiracle at the first day of Bombyx larval wandering stage. We injected $0.4-0.5 \mu \mathrm{g}$ of dsptl at the ratio of 1:1 mix ptl1and ptl2 final instar larvae of Tribolium castaneum. To knockdown CPH28 expression in the silkworm pupal stage, the siRNA sites 5'-GCAGCAAUUGUUCGCACAATT-3' and 5'-GGAAGCUUUACAUUCGGUUTT-3' (GenePharma) for CPH28 were designed. Ten $\mu$ l of siRNA $1 \mu \mathrm{g} / \mu \mathrm{l}$ was injected from the breathing-valve into the wing disc on the $4^{\text {th }}$ day of the pupal stage. In addition, after injection, all insects were reared in a suitable living environment until analysis.

Down-regulation of Antp in Drosophila wings We used the Gal4/UAS system to 
knockdown Antp gene expression in Drosophila wings. We crossed the UAS-Antp ${ }^{R N A i}$ males with nub-gal4 virgin females and then incubated them at $25^{\circ} \mathrm{C}$ on a yeast/saccharose medium. The wing phenotypes of $\mathrm{F} 1$ adults were observed.

CRISPR/Cas9-mediated Antp Knockout in Bombyx. The sgRNAs for knocking out Antp was designed by http://crispr.dbcls.jp/ and synthesized using the RiboMAXTM Large Scale RNA Production System T7 kit (Promega). Cas9 protein was purchased from Invitrogen (Thermo). The four sgRNAs and the Cas9 protein were mixed at a dose of $500 \mathrm{ng} / \mathrm{ul}$. The mixture was incubated for $15 \mathrm{~min}$ at $37^{\circ} \mathrm{C}$ to produce a ribonuclearprotein complex (RNP) and micro-injected into the silkworm embryos within $2 \mathrm{~h}$ post oviposition. The injected embryos were incubated at $25^{\circ} \mathrm{C}$ and $>90 \%$ relative humidity until they hatched. Genomic DNA of adult wings was extracted using the DNAzol (Takara) according to the manufacturer protocol. The target region was amplified using site-specific primers (Table S1). PCR products were checked by PAGE gel and sequencing approach. Related promoters are listed in Table S1. These sgRNAs synthesized in vitro were mixed with Cas9 protein and micro-injected into preblastoderm embryos of the DaZao strain.

ELISA. ELISA was used to calibrate the ecdysteroid titer in wing disc of WT and Antp mutants. Silkworm wing discs were collected from 50 pupae, and the pooled sample homogenized in methanol. The homogenate was centrifuged and we evaporated the supernatant at $55^{\circ} \mathrm{C}$. The solid matter remaining was redissoved in 1 mL EIA buffer (Cayman Chemical) for 20E measurement and $1 \mathrm{~mL}$ sample diluents (BIOHJ) for ecdysone measurement, respectively. Ecdysteroid titers were assayed by an ELISA kit according to manufacturer instructions (Cayman Chemical or BIOHJ). Absorbance was measured at $414 \mathrm{~nm}$ for Cayman kit or $450 \mathrm{~nm}$ for BIOHJ kit on a BioTek H1 microplate reader.

20E Application. For 20E treatment in Bombyx and BmN cells, 20E (Adooq) was dissolved in DMSO and then diluted to the experimental concentrations with deionized distilled water. The final concentration of DMSO was $0.1 \%(\mathrm{v} / \mathrm{v})$ in water. A total of $4 \mu \mathrm{g} 20 \mathrm{E}$ was injected into larvae at the mesothoracic region on the 1 st day of the larval wandering stage. An equal volume of DMSO at a final concentration of $0.1 \%(\mathrm{v} / \mathrm{v})$ was used as the control. After $24 \mathrm{~h}$, the wing discs were dissected in TRK lysis buffer (Omega). Five- $\mu \mathrm{m}$ 20E were applied to BmN cells for $24 \mathrm{~h}$ and then collected. An equal volume of DMSO was used as the control.

Dual Luciferase Assay. The different lengths of shade, CPH28, and Antp promoters were subcloned into the pGL3-basic vector (Promega). The ORF of red fluorescent protein gene (RFP)-fused Antp was inserted into a pIZ/V5-His vector (Invitrogen) driven by the OpIE2 promoter. Different truncated promoters of pGL3-basic vector were co-transfected with pIZ/V5-His-Antp or treated with $20 \mathrm{E}$ at a concentration of 5 uM. After approximately 24 or $48 \mathrm{~h}$ transient transfection, dual-luciferase activities were measured using the Dual-Glo Luciferase Assay Kit (Promega). A pRL-TK vector 
containing the Renilla luciferase gene was used as an internal control.

EMSA. Recombinant Antp nuclear proteins were extracted from E. coli strain BL21 (DE3) competent cells (TransGen). The potential Antp binding sites of the shade and $\mathrm{CPH} 28$ promoters were predicted by the GENOMATIX system (http://www.genomatix.de/solutions/index.html) and JASPAR CORE (http://jaspar.genereg.net/). The DNA oligonucleotides containing Antp binding sites were labeled with biotin at the 5'-end and annealed to generate probes. EMSA experiments were conducted according to manufacturer instructions for the EMSA/Gel-Shift Kit (Beyotime). The binding reactions were performed with $4 \mu \mathrm{g}$ recombinant Antp protein and different amounts of biotin-labeled probes (10 pmol, 20 pmol, $40 \mathrm{pmol}$ ) for $30 \mathrm{~min}$ at room temperature. For competition assays, $40 \mathrm{pmol}$ unlabeled competitor probes were added to the reaction mixture. These samples were electrophoresed on $5 \%$ polyacrylamide gels in $0.5 \times \mathrm{TBE}$ at room temperature. The total probes are listed in S1 Table.

ChIP Assay. To further detect the effects of Antp on the activity of the shade and CPH 28 promoters, the ChIP assay was performed following kit instructions (GST). $\mathrm{BmN}$ cells were transfected with a Flag-Antp expression vector and harvested at $48 \mathrm{~h}$. These cells were fixed with $37 \%$ formaldehyde, and then DNA containing proteins were sonicated to obtain 200-1000 bp length DNA fragments. The immunoprecipitation reactions were enriched with $1 \mu \mathrm{g}$ antibody against Flag or IgG. The precipitated DNA and input were used for PCR analysis. The primers used for amplifying the sequences containing potential Antp binding sites are listed in S1 Table.

Statistical Analysis. Statistical analyses were performed using GraphPad Prism 7 (GraphPad Software). The data are presented as the mean \pm standard error (SE). The differences between two sets of data were analyzed with Student's t-test. A value of $\mathrm{P}<0.05$ was considered statistically significant; $* \mathrm{P}<0.05, * * \mathrm{P}<0.01$, and $* * * \mathrm{P}<$ 0.001 .

\section{Acknowledgments}

This work was supported by the National Natural Science Foundation of China [No. U20A2058, No. 31830094]. AM acknowledges support from the National Research Foundation Singapore Investigatorship award NRF-NRFI05-2019-0006.

\section{Author contributions}

X. T. and C. F. designed the project. C. F., Y. X., and T. S. performed the experiment. C. F., A. M., and X. T. wrote the manuscript.

\section{Competing interests}


The authors declare no competing financial interests.

\section{References}

1. Mallo M, Alonso CR. The regulation of Hox gene expression during animal development. Development. 2013;140(19):3951- 63.

2. Carroll SB, Weatherb Ee SD, Langeland JA. Homeotic genes and the regulation and evolution of insect wingnumber. Nature. 1995;375(6526):58-61.

3. Tomoyasu Y, Wheeler SR, Denell RE. Ultrabithorax is required for membranous wing identity in the beetle Tribolium castaneum. Nature. 2005;433(7026):643-7.

4. Deutsch J. Hox and wings. Bioessays. 2005;27(7):673-5.

5. Struhl G. Genes controlling segmental specification in the Drosophila thorax. Proc. Natl. Acad. Sci. USA 79, 7380-7384. Proceedings of the National Academy of Science. 1983;79(23):7380-4.

6. Roch F, Akam M. Ultrabithorax and the control of cell morphology in Drosophila halteres. Development. 2000;127(1):97-107.

7. Tomoyasu, Yoshinori. Ultrabithorax and the evolution of insect forewing/hindwing differentiation. Curr. Opin. Insect Sci. 2017;19, 8-15.

8. Liu F, Li X, Zhao M, Guo M, Han K, Dong X, et al. Ultrabithorax is a key regulator for the dimorphism of wings, a main cause for the outbreak of planthoppers in rice. Natl. Sci. Rev.2020;(7):7.

9. Weatherbee SD, Halder G, Kim, J, Hudson A, Carroll S. Ultrabithorax regulates genes at several levels of the wing-patterning hierarchy to shape the development of the Drosophila haltere. Genes Dev. 1998;12(10):1474-82

10. Weatherbee SD, Nijhout HF, Grunert LW, Halder G, Galant R, Selegue J, et al. Ultrabithorax function in butterfly wings and the evolution of insect wing patterns. Current Biology Cb. 1999;9(3):109-15.

11. Lewis EB. A gene complex controlling segmentation in Drosophila. Nature. 1978; 276(5688):565-70.

12. Pavlopoulos A, Akam M. Hox gene Ultrabithorax regulates distinct sets of target genes at successive stages of Drosophila haltere morphogenesis. Proc. Natl. Acad. Sci. USA. 2011;108(7):2855-60.

13. Carroll SB, Weatherb Ee SD, Langeland JA. Homeotic genes and the regulation and evolution of insect wingnumber. Nature.1995;375(6526):58-61.

14. Nagata T, Suzuki Y, Ueno K, Kokubo H, Xu X, Hui C, et al. Developmental expression of the Bombyx Antennapedia homologue and homeotic changes in the $\mathrm{Nc}$ mutant. Gene cells. 1996;1(6):555-68.

15. Chen P, Tong XL, Li DD, Fu MY, He SZ, Hu H, et al. Antennapedia is involved in the development of thoracic legs and segmentation in the silkworm, Bombyx mori. Heredity. 2013;111(3):182-8.

16. Denell RE, Hummels KR, Wakimoto BT, Kaufman TC. Developmental studies of lethality associated with the Antennapedia gene complex in Drosophila melanogaster. Dev Biol. 1981;81(1):43-50. 
17. Wakimoto BT, Kaufman TC. Analysis of larval segmentation in lethal genotypes associated with the antennapedia gene complex in Drosophila melanogaster. Dev. Biol. 1981;81(1):51-64.

18. Mizoguchi A, Ohashi Y, Hosoda K, Ishibashi J, Kataoka H. Developmental profile of the changes in the prothoracicotropic hormone titer in hemolymph of the silkworm Bombyx mori: correlation with ecdysteroid secretion. Insect Biochem Mol Biol. 2001;31(4):349-58.

19. Fujiwara H, Hojyo T. Developmental profiles of wing imaginal discs of flügellos(fl), a wingless mutant of the silkworm, Bombyx mori. Genes Evol. 1997;207(1):12-8.

20. D'Avino PP, Thummel CS. The ecdysone regulatory pathway controls wing morphogenesis and integrin expression during Drosophila metamorphosis. Dev. Biol. 2000;220(2):211-24.

21. Schubiger M, Truman JW. The RXR ortholog USP suppresses early metamorphic processes in Drosophila in the absence of ecdysteroids. Development 2000;127(6):1151-9.

22. Kawasaki H, Kiguchi K, Agui N, Iwashita Y. Ecdysteroid titer and Wing Development during the Pupal-Adult Transformation of Bombyx mori. ZOOL Sci. 1986;3:301-8.

23. Futahashi R, Okamoto S, Kawasaki H, Zhong YS, Iwanaga M, Mita K, et al. Genome-wide identification of cuticular protein genes in the silkworm, Bombyx mori. Insect Biochem Mol Biol. 2008;38(12):1138-46.

24. Riddiford LM, Cherbas P, Truman JW. Ecdysone receptors and their biological actions. Vitam Horm. 2000;60:1-73.

25. Petryk A, Warren JT, Marques G, Jarcho MP, Gilbert LI, Kahler J, et al. Shade is the Drosophila P450 enzyme that mediates the hydroxylation of ecdysone to the steroid insect molting hormone 20-hydroxyecdysone. Proc. Natl. Acad. Sci. USA. 2003;100(24):13773-8.

26. Wang HB, Moriyama M, Iwanaga M, Kawasaki H. Ecdysone directly and indirectly regulates a cuticle protein gene, BMWCP10, in the wing disc of Bombyx mori. Insect Biochem Mol Biol. 2010;40(6):453-459.

27. Cifuentes FJ, Garciabellido A. Proximo-distal specification in the wing disc of Drosophila by the nubbin gene. Proc. Natl. Acad. Sci. USA.1997;94(21):11405-10.

28. Matsuoka Y, Monteiro A. Hox genes are essential for the development of eyespots in Bicyclus anynana butterflies. Genetics. 2021;217(1):1-9.

29. Hombría CG. Butterfly eyespot serial homology: enter the Hox genes. BMC Biol. 2011;9:26.

30. Rewitz KF, Rybczynski R, Warren JT, Gilbert LI. Developmental expression of Manduca shade, the P450 mediating the final step in molting hormone synthesis. Mol Cell Endocrinol. 2006;247(1-2):166-74.

31. Shahin R, Iwanaga M, Kawasaki H. Expression profiles of cuticular protein genes in wing tissues during pupal to adult stages and the deduced adult cuticular structure of Bombyx mori. Gene.2018;646:181-194.

32. Gilbert LI, Warren JT. A molecular genetic approach to the biosynthesis of the 
520 insect steroid molting hormone. Vitam Horm. 2005;73:31-57.

521 33. Monier B, Astier M, Sémériva M, Perrin L. Steroid-dependent modification of 522 Hox function drives myocyte reprogramming in the Drosophila heart. Development. $523 \quad 2005 ; 132(23): 5283-93$.

524 34. Ou J, Deng HM, Zheng SC, Huang LH, Feng QL, Liu L. Transcriptomic analysis 525 of developmental features of Bombyx mori wing disc during metamorphosis. BMC 526 Genomics. 2014;15(1):820. 
A

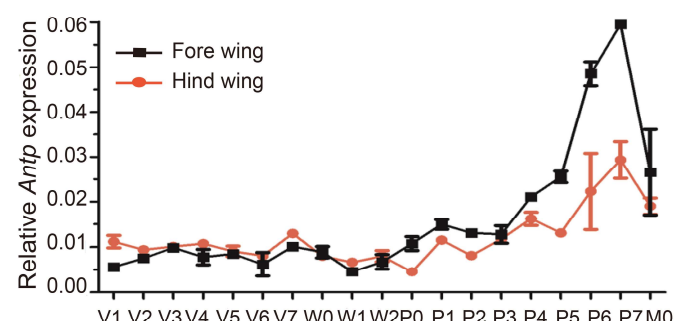

V1 V2 V3V4 V5 V6 V7 Wo W1 W2 PPO P1 P2 P3 P4 P5 P6 P7MO

D

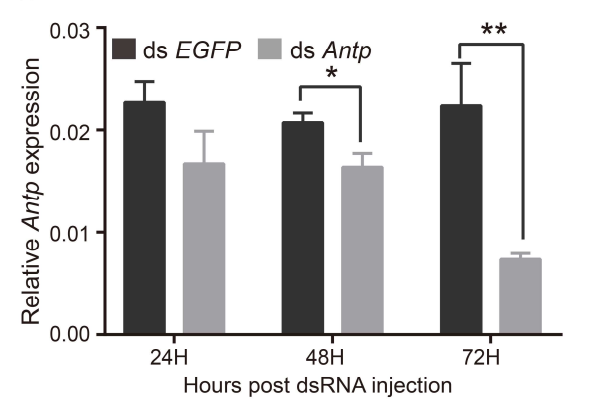

E

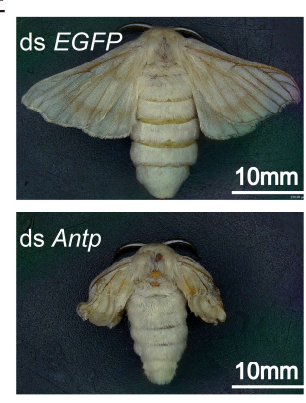

B

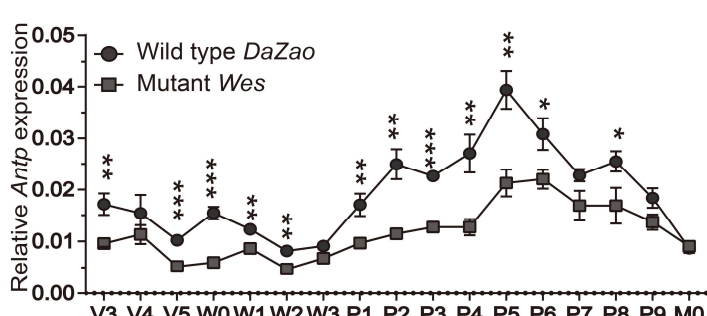

C

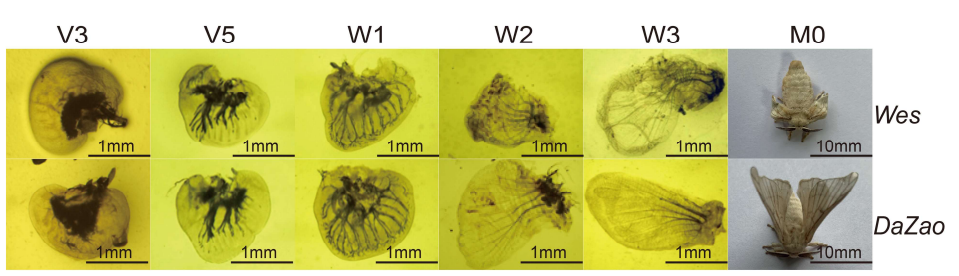

F Antp gene

(2)
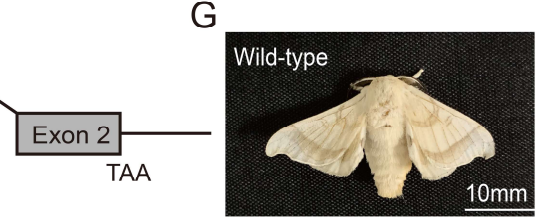

Target site 1 ATGGCTGCGATCAGCAGCTCAGG

Target site 2 CTGCGATCAGCAGCTCAGGCCGG

Target site 3 GCCGGCACAGCATCACTACCCGG

Target site 4 CGCCTGGAATGCCCTACCCCAGG

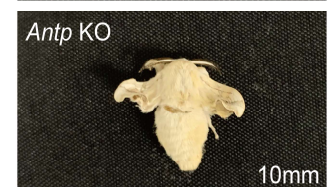

$\mathrm{H}$

Target site 1 Target site $2 \quad$ Target site 3

Target site 4

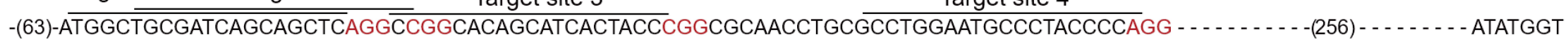

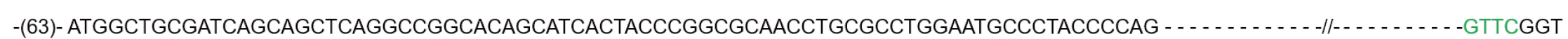

-(63)- AAAAAAAACGCGTAATATTTCTCGAACAATCTTATCTCACCC - $\mathbf{-}-\mathbf{n}$

-(63)- ATTGATTTAGCGGCCGCGAATTGGCCCTTGTCACGAACATCAACAGGCACACACACACACACACATATGCATGCACACGTACACACTCACACACGTATA-//-ATATGGT

531 Fig 1. Antp is essential for wing development in B. mori. (A) Temporal expression pattern of Antp in wild-type (DaZao) forewing and 
hindwing discs by qRT-PCR. (B) Expression profiles of Antp in the wing discs of wild-type and mutant (Wes) lines from larvae to adult stages. (C) Phenotype of the wing discs in wild-type DaZao and Wes $\left(A n t p^{+-}\right)$mutants over different time points. (D) Relative Antp expression levels of dsRNA-treated larvae at $24 \mathrm{~h}, 48 \mathrm{~h}$, and $72 \mathrm{~h}$ after dsRNA treatments. Animals injected with dsEGFP served as controls. (E) Wing phenotype of dsRNA-treated silkworm adults. (F) Genomic structure of Antp. The single guide RNA (sgRNA) target sequence is in black font and the protospacer adjacent motif (PAM) sequence is in red font. The red arrows mark the sgRNA targets on the Antp gene. F and R indicate the approximate locations of the amplification primers. (G) Representative phenotypes of wild-type (top) and mutated (bottom) insects, with smaller and abnormal wings. (H) Mutated sequences of crispant individuals. The wild-type sequence, showed above the mutant sequences, is in black and the PAM sequence is in red font. The size of indels is shown to the right of the sequence. Inserted sequenced are in green font. For all graphs, $\mathrm{V}$ is the 5th instar larvae, V1-V7 means days $1-7$ of the 5th instar larvae; W is the wandering larval stage, W0-W3 indicates days $0-3$ of the wandering larval stage; P, the pupal stage, P0-P9 indicates days of 0-9 of the pupal stage; M0, newly emerged adult. All experimental data shown are means $\pm \mathrm{SE}(\mathrm{n}=3)$. Asterisks indicate significant differences with a two-tailed t-test: $* \mathrm{P}<0.05, * * \mathrm{P}<0.01, * * * \mathrm{P}<0.001$. 


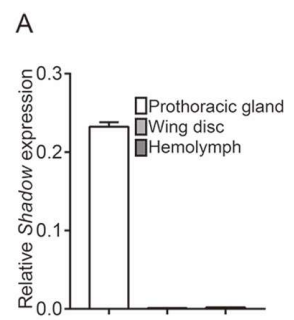

$\mathrm{F}$
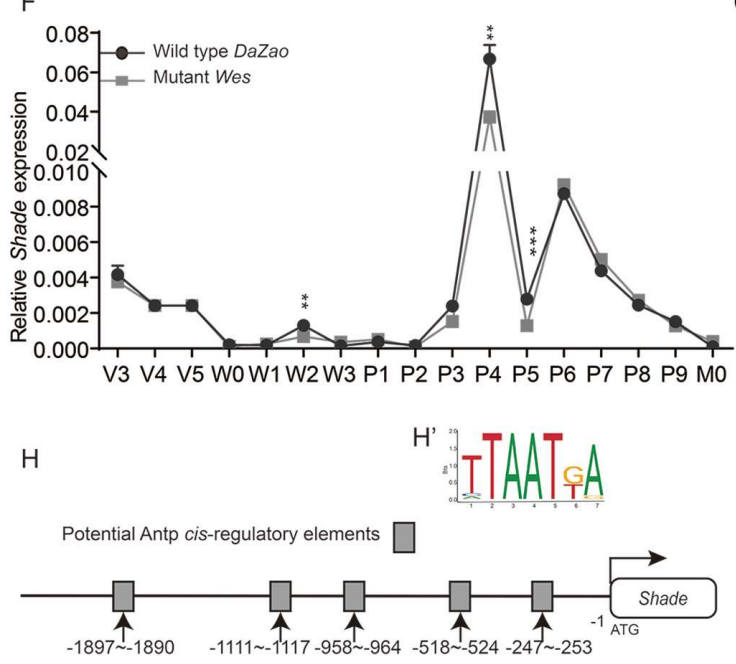

J

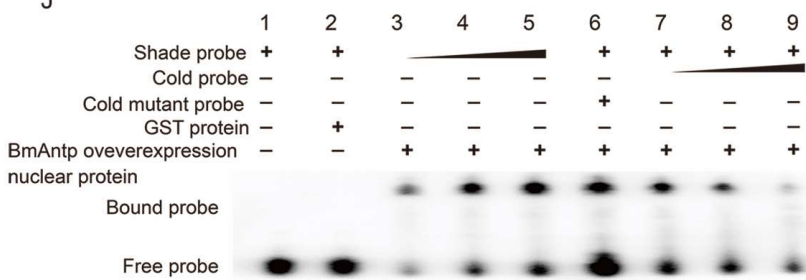

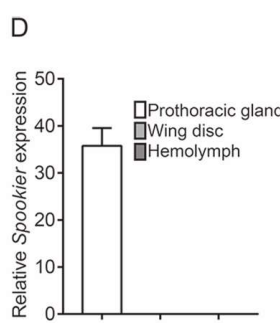
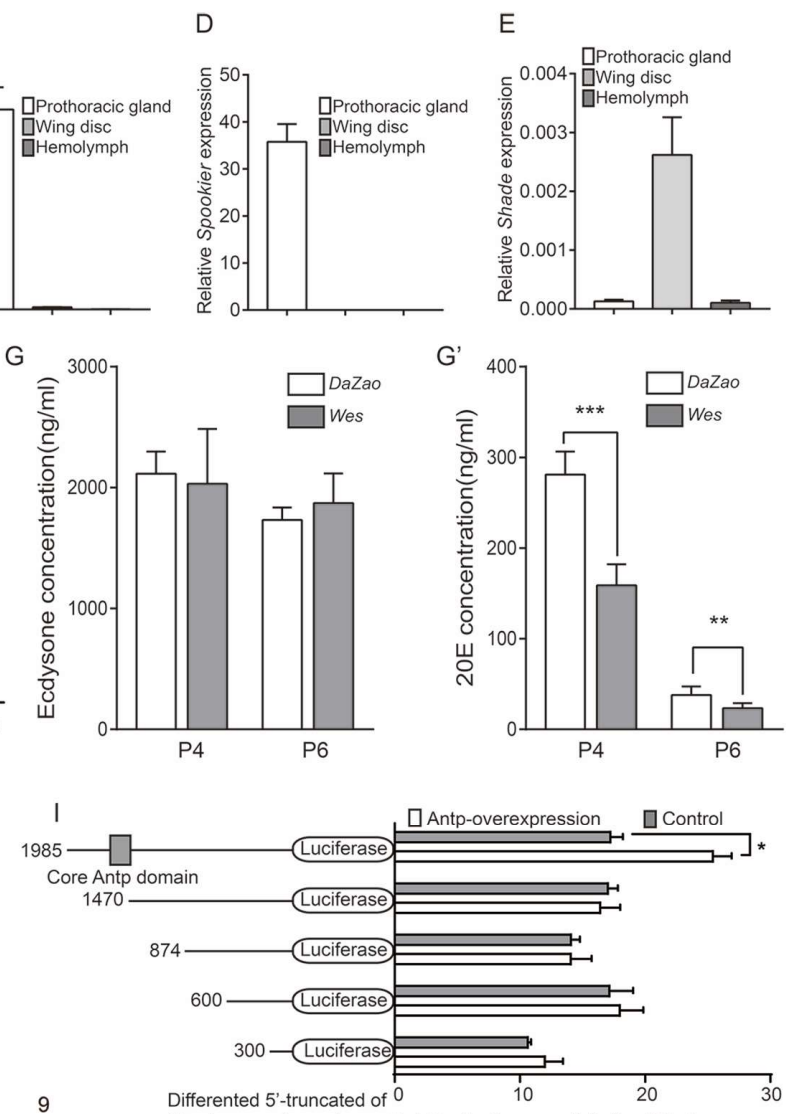

Shade promoter regions Relative luciferase activity(Luc/Rluc)

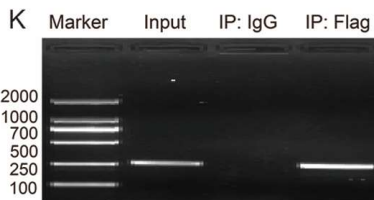

Fig 2. Antp induces $20 \mathrm{E}$ synthesis in the wing tissue by directly binding to the shade promoter. (A-E) Relative expression of five ecdysteriod enzyme genes in the prothoracic gland, hemolymph, and wing disc. (F) mRNA levels of shade were detected by qRT-PCR from the 5th instar larval stage to the adult stage. (G and G') The titers of ecdysone $(\mathrm{G})$ and 20E (G') in Bombyx wing discs of WT DaZao and Wes mutants $\left(A n t p^{+-}\right)$at P4 and P6. (H) Location of the five potential Antp binding sites in the shade promoter. (H') Classic Antp binding motif. (I) The effect of different truncations of the shade promoter on luciferase activity when Antp is overexpressed in BmN cells. (J) EMSA confirmed that the recombined Antp proteins bind to the nt -1897--1890 region in the Shade promoter. Coincubating nucleoproteins from Escherichia coli strain BL21 (DE3) competent cells overexpressing GST with labeled Antp probes resulted in loss of the binding band. Purified recombinant BmAntp protein could bind to the biotinylated probes in a dose-dependent manner (lanes 3-5), and this binding could be competitively suppressed by unlabeled probe (lane 6). The unlabeled probe with mutation in the core-binding motif of BmAntp could not compete for BmAntp binding to biotinylated probes (lanes 7-9). We further validated the direct regulation of BmAntp on shade transcription through in vivo ChIP-PCR following the BmN cells which were overexpression of FLAG-tagged BmAntp. (K) 
561 ChIP-PCR assay of the direct binding of Antp to the shade promoter in BmN cells

562 with Antp-Flag overexpression. Specific primers covering Antp binding sites of the 3

563 Shade promoter were used. Comparing with nonspecific IgG antibody, used as a

564 negative control, the antibody against FLAG can specifically immunoprecipitate the

565 DNA regions including -1985 to -1470 of the Shade promoter. All experimental data

566 shown are means $\pm \mathrm{SE}(\mathrm{n}=3)$. Asterisks indicate significant differences with a two-tailed t-test: $* \mathrm{P}<0.05, * * \mathrm{P}<0.01$, and $* * * \mathrm{P}<0.001$. 
BmAntp promoter

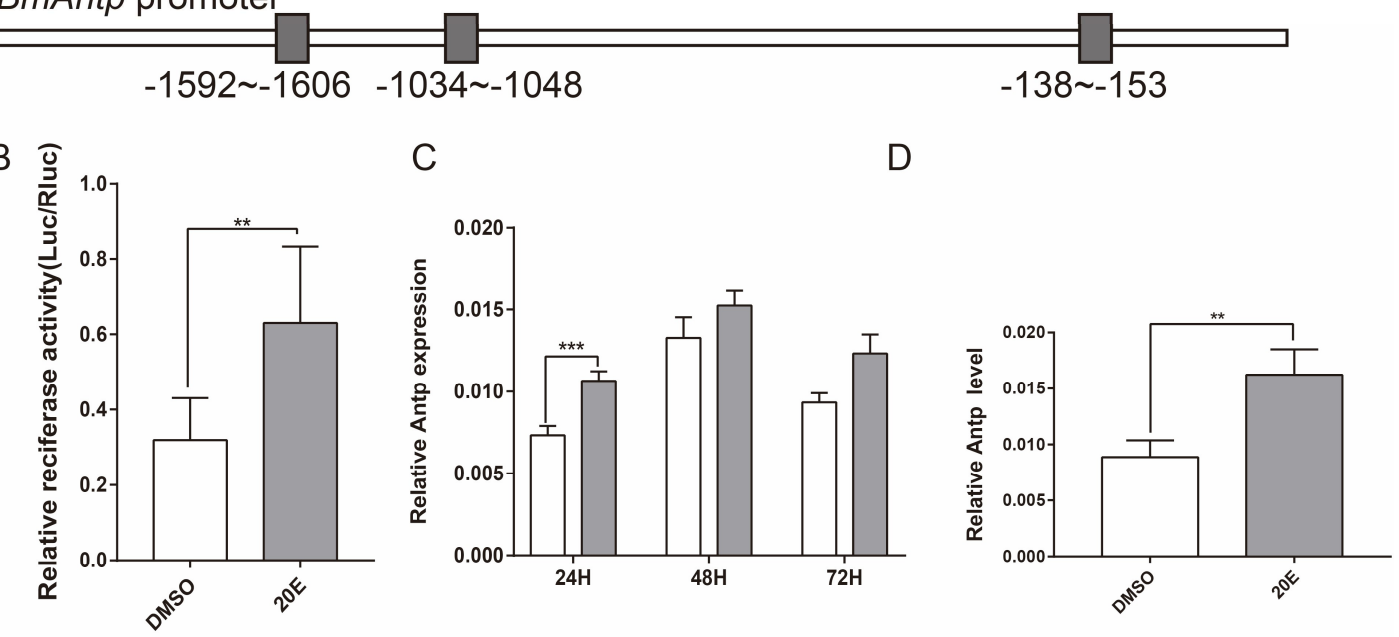

Fig 3. 20E induced expression of Antp. (A) Location of potential Antp binding sites in the Antp promoter. (B) Effects of $20 \mathrm{E}$ treatment on the luciferase activity driven by the Antp promoter. (C and D) Levels of Antp expression increase in BmN cells (C) and wing discs (D) after 20E treatment. All of the experimental data shown are means $\pm \mathrm{SE}(\mathrm{n}=3)$. Asterisks indicate significant differences with a two-tailed t-test: 

A
B
C
D
E
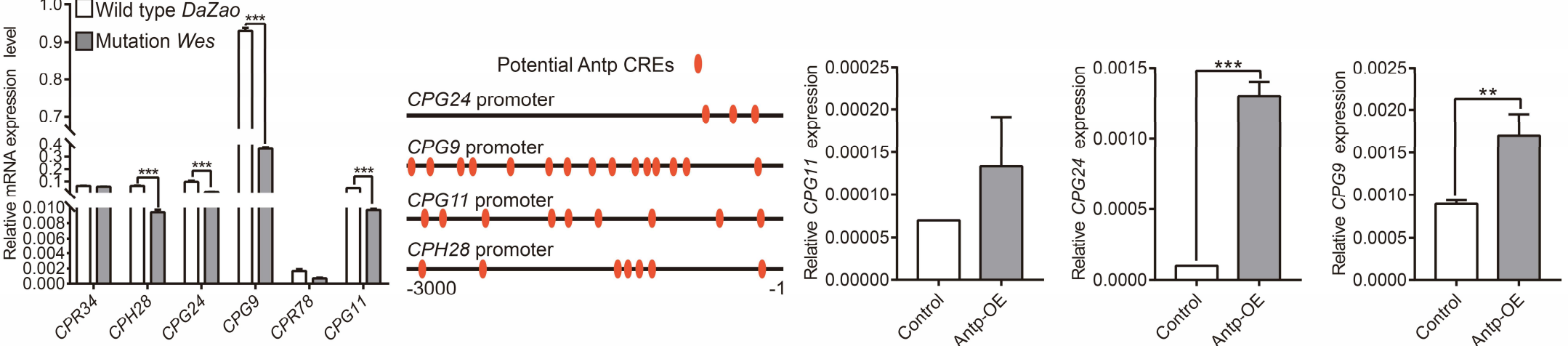

F

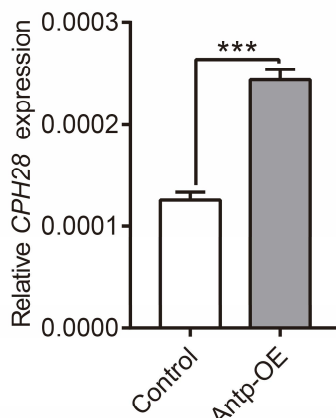

G
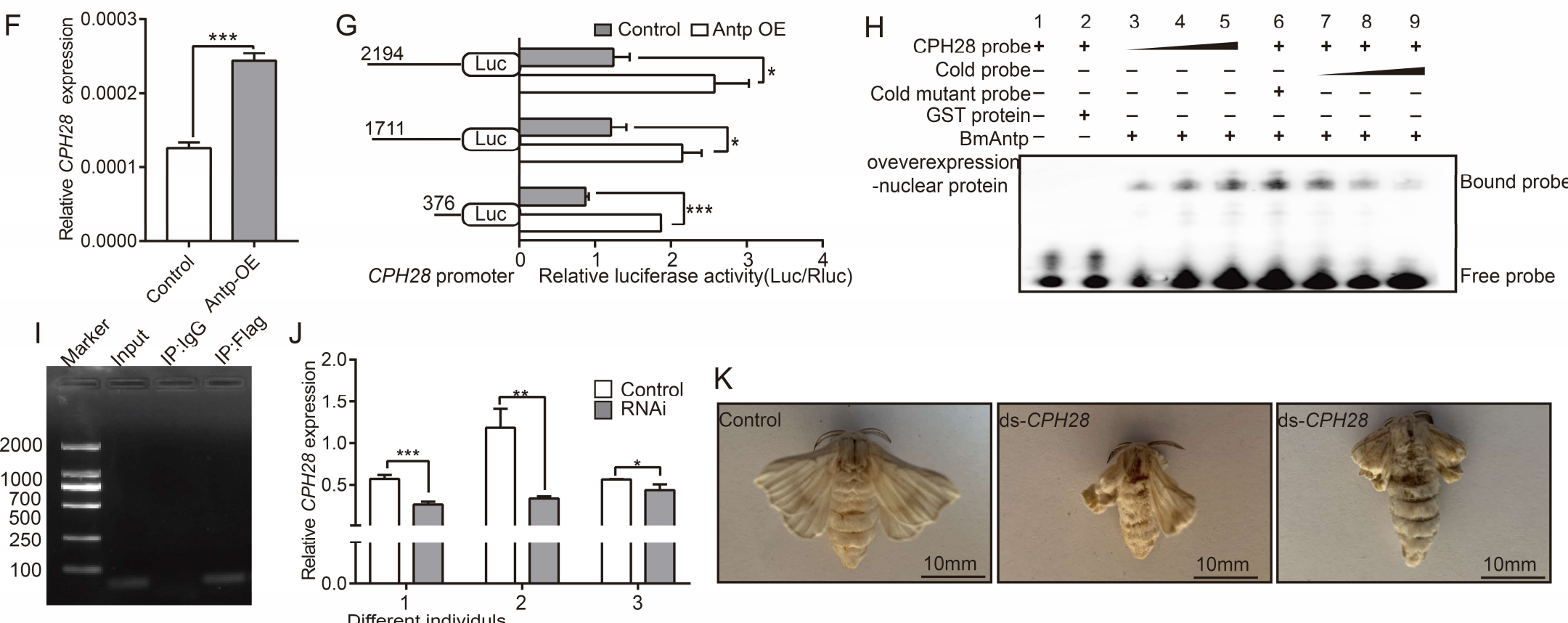

K

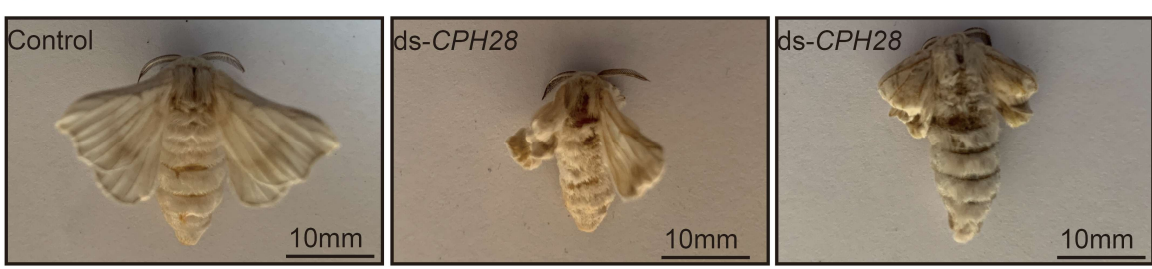

Different individuls 
Fig 4. Antp regulates the expression of cuticular protein genes essential for wing development. (A) mRNA levels of cuticular protein genes in wing discs of DaZao and Wes (Antp $\left.{ }^{+/}\right)$at P5. (B) Schematic of the potential Antp CREs in the promoters of cuticular protein genes. (C-F) Relative cuticular protein genes expression detected in Antp overexpression BmN cells. (G) Antp increased luciferase activity driven by different truncations of the $\mathrm{CPH} 28$ promoter. (H) Electrophoretic mobility shift assay (EMSA) of the binding nuclear proteins extracted from Antp-overexpressing Escherichia coli strain BL21 (DE3) competent cells with the Antp binding motif. Co-incubating nucleoproteins from E. coli strain BL21 (DE3) competent cells overexpressing glutathione S-transferase (GST) with labeled Antp probes results in loss of the binding band. The binding signal between recombinant GST-BmAntp protein and Antp binding motif probe was gradually enhanced with increased probe levels (lanes 3-5). (I) ChIP-PCR assay shows that Antp binds directly to Antp binding motifs present in the CPH 28 promoter in BmN cells. A Flag tag was fused to BmAntp and an anti-Flag tag antibody was used in the ChIP assay. The cells were transfected with recombinant plasmid Flag-BmAntp, and then the cells were collected for ChIP assay $48 \mathrm{~h}$ post-transfection. The results showed that the anti-Flag antibodies, but not IgG (a negative control), precipitated DNA containing the Antp binding motifs in the cells transfected with the Flag-BmAntp expressing plasmid. (J) qPCR analyses of $\mathrm{CPH} 28$ expression in wing discs of different individuals, $48 \mathrm{~h}$ after knock down of $C P H 28$ and of a control sequence (containing the scrambled siRNA sequence). (K) Comparisons of adult wing morphology after dsRNA injections. All experimental data shown are means $\pm \mathrm{SE}(\mathrm{n}=3)$. Asterisks indicate significant differences with a two-tailed t-test: $* \mathrm{P}<0.05, * * \mathrm{P}<0.01$, and $* * * \mathrm{P}<0.001$. 
A
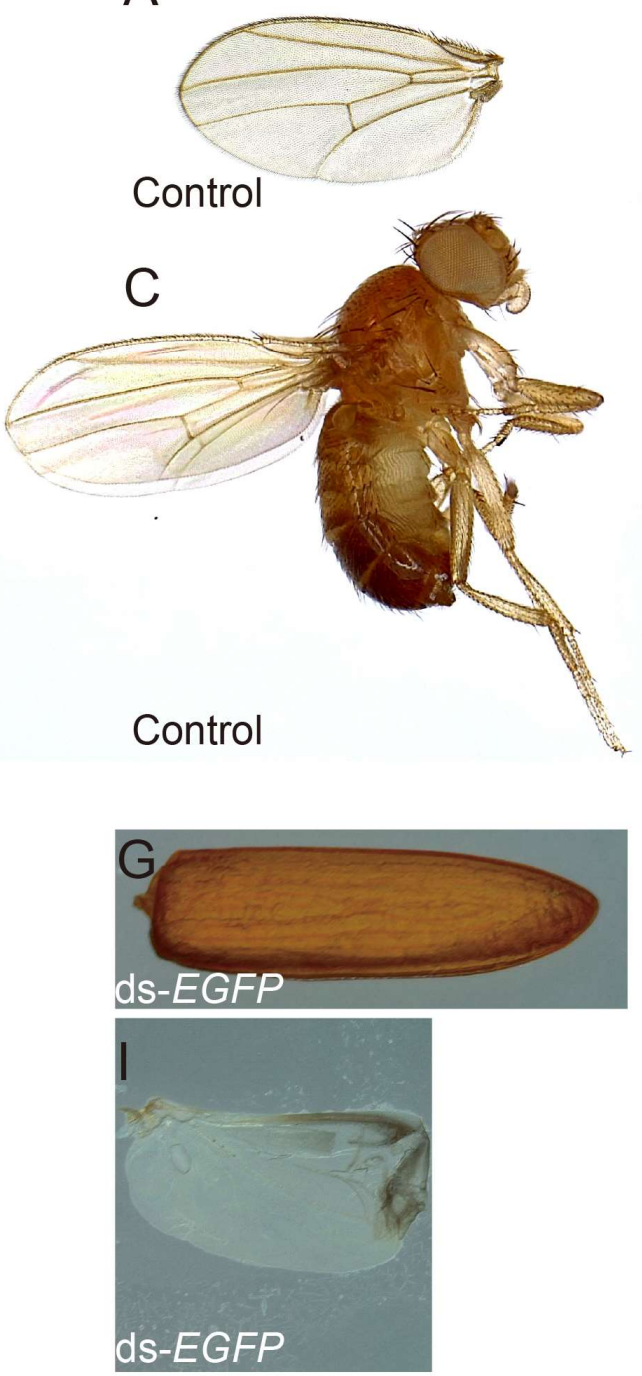

B

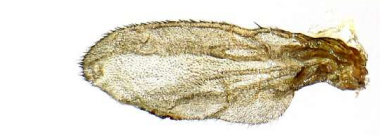

RNAi Antp

D

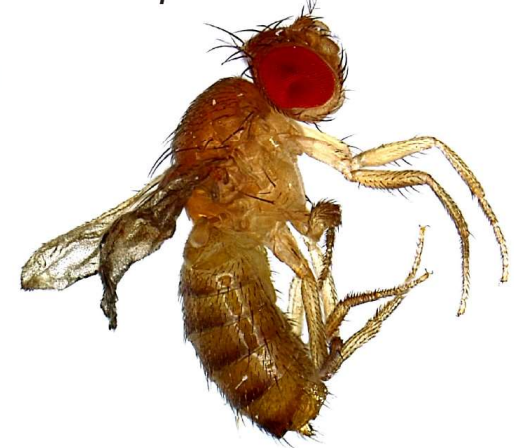

RNAi Antp
E

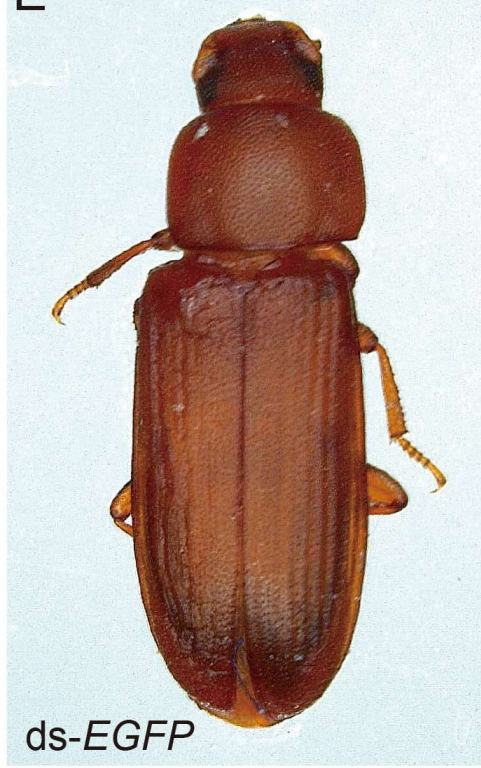

F

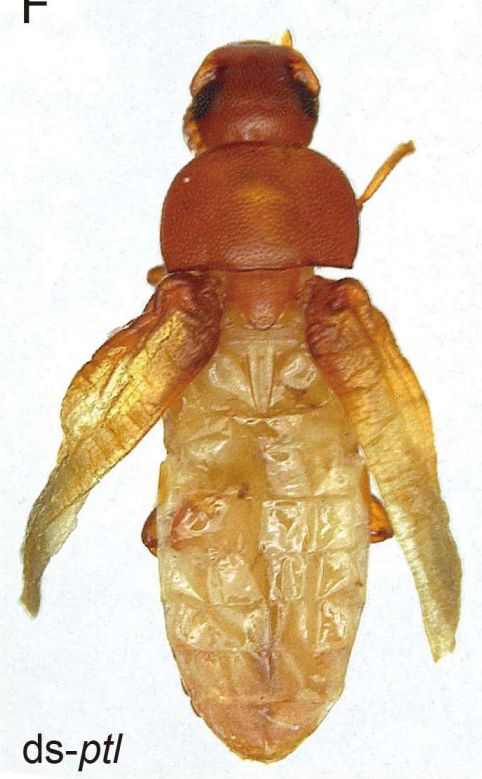

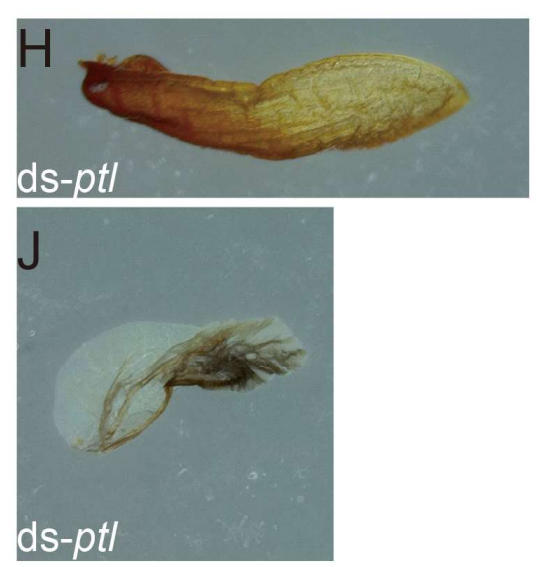
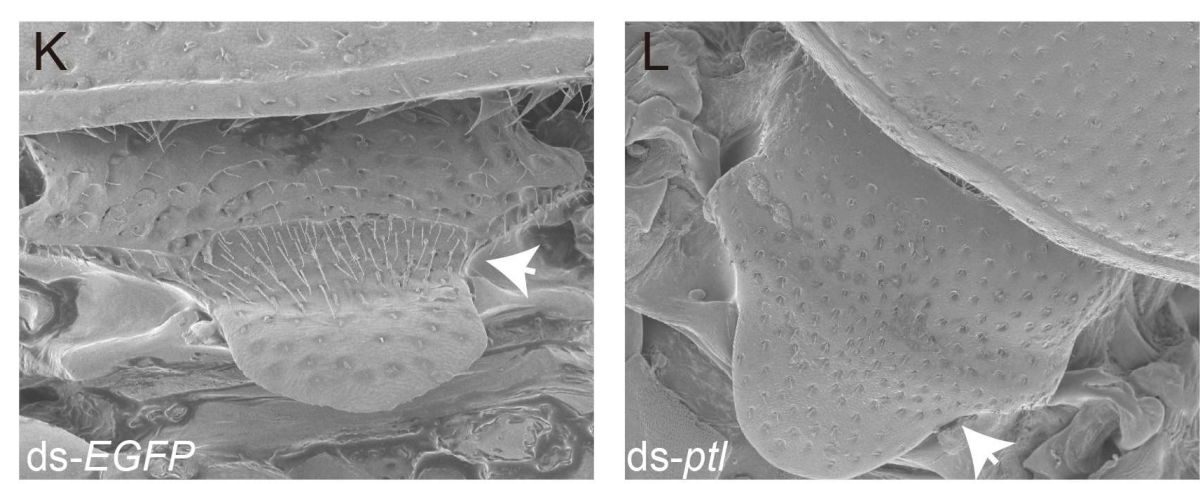
Fig 5. Antp is essential for wing development in Drosophila and Tribolium. (A and B) Adult Drosophila wings from control (A) and Antp RNAi (B) treated individuals. (C and D) Adult Drosophila of control (C) and Antp RNAi (D) treated individuals. (E and F) ptl RNAi leads to reduction of elytra and hindwings in Tribolium adults. (E) ds-EGFP. (F) ds-ptl. (G and I) The elytron (G) and hindwing (I) from a ds-EGFP treated individual. ( $\mathrm{H}$ and $\mathrm{J}$ ) The elytron $(\mathrm{H})$ and hindwing $(\mathrm{J})$ from a ds-ptl treated individual. (K and L) ptl RNAi leads to a uniform mesonotum (white arrow in $\mathrm{K}$ and $L$ ). (K) ds-EGFP. (L) ds-ptl.

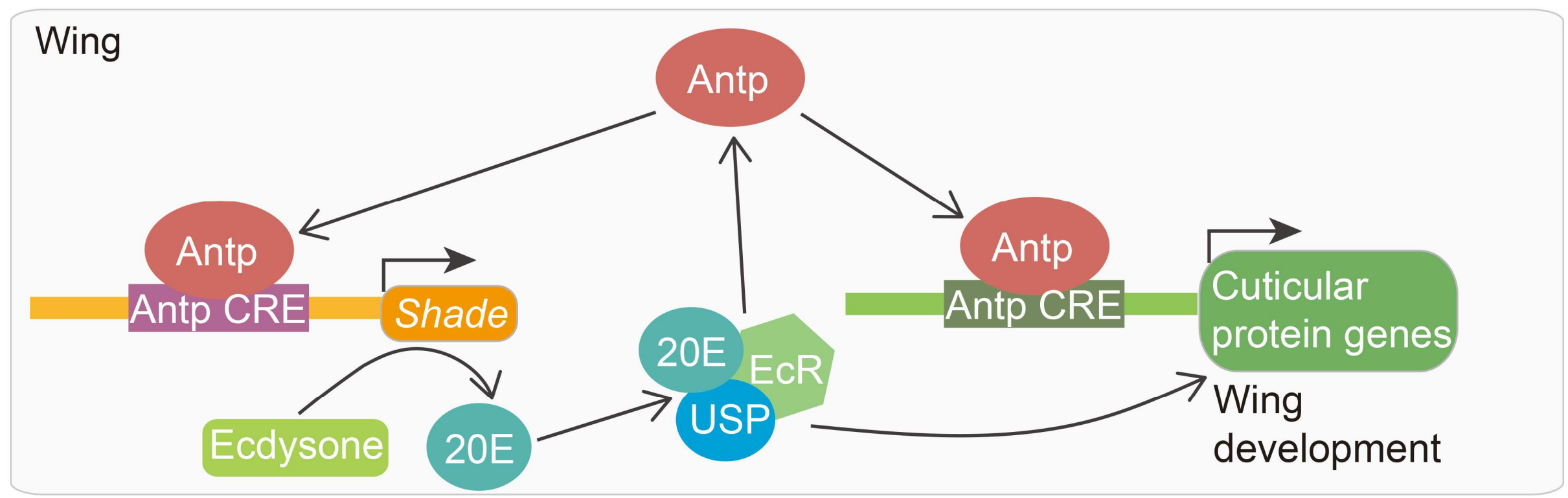

Fig 6. Proposed model on how Antp regulates wing development in B. mori. The Hox gene Antp plays an essential role in wing development. It does this by directly enhancing transcription of the steroidogenic enzyme gene shade in wings and, thus, controlling the synthesis of an essential growth hormone, $20 \mathrm{E}$, directly in the wing tissue. In turn 20E signaling upregulates Antp expression. Antp also directly regulates the expression of critical cuticular protein genes in both forewings and hindwings. 